\title{
The Incidence of Carbapenem Resistance in Klebsiella pneumoniae Subspecies pneumoniae (CRKP) and Antibiotic Susceptibility Profile of Klebsiella pneumoniae Subspecies pneumoniae strains
}

\author{
Priyanka Singh* \\ Department of Microbiology, RKDF Medical College and Research Center, Jatkhedi \\ Hosangabad Road, Bhopal-462026, India \\ *Corresponding author
}

\begin{tabular}{|l|}
\hline Ke y w o r d s \\
$\begin{array}{l}\text { Klebsiella } \\
\text { pneumoniae, CRKP }\end{array}$ \\
\hline Article Info \\
\hline $\begin{array}{l}\text { Accepted: } \\
\text { 06 December } 2017 \\
\text { Available Online: } \\
\text { 10 January } 2018\end{array}$ \\
\hline
\end{tabular}

\section{Introduction}

Carbapenamases represent the most versatile family of $\beta$ - lactamases with broad spectrum of activity unrivaled by other $\beta$-lactamhydrolysing enzymes. They were known as "carbapenamases" as many of them recognize all hydrolysable $\beta$ - lactams and most are not inhibited by commercially available $\beta$ - lactamase inhibitors (Walther-Rasmuseen and Hoiby, 2006). Carbapenems are often used as an antibiotic of last resort for treating serious infections caused by multidrug resistant organism. Reduced susceptibility to any carbapenem can be used as screening of carbapenamase. In recent years, carbapenem resistance has emerged in Klebsiella pneumoniae isolates due to acquisition of 
carbapenamase genes. The production of carbapenamase enzyme has direct carbapenem hydrolyzing activity. Recently Carbapenem resistant pose a real threat to Medical fraternity as the increased frequency with which Enterobacteriaceae cause infection and the increased mortality associated with infections caused by carbapenem resistance.

Metallo betalactamases (MBL) has gained importance in recent years as MBL genes located on integron that resides on mobile genetic elements and can disseminates in hospital Many phenotypic methods have been proposed by CLSI.

Carbapenemases belongs to two major molecular families distinguished by the hydrolytic mechanism at the active sites. Carbapenamases are classified according to their amino acid sequences: Ambler class A (Serine carbapenemases); Class B (Metallo $\beta$ lactamases); and Class D (OXA carbapenemase).

\section{Classification of carbapenemases}

Carbapenemases are divided into two groups according to their active sites:

Serine carbapenemases belonging to the class A Penicillinases and class D Oxacillinases, which contain a serine in the active site and can be inactivated by $\beta$-lactamase inhibitors, including clavulanic acid and tazobactam.

Metallo- $\beta$-lactamases belonging to the class B carbapenemases, which contain one or more zinc atoms at the active sites, allowing them to hydrolyse the bicyclic $\beta$-lactam ring. These enzymes are inhibited by EDTA. (Queenan and Bush K. Carbapenemase, 2007)

Carbapenemases belongs to two major molecular families distinguished by the hydrolytic mechanism at the active sites.
Carbapenamases are classified according to their amino acid sequences

Ambler class A (Serine carbapenemases); Class B (Metallo $\beta$ lactamases); and Class D (OXA carbapenemase).

\section{Aims and objectives}

To study Klebsiella pneumoniae subspecies pneumoniae with special reference to carbapenemases production isolated from different clinical specimens in the Department of Microbiology, Jawaharlal Nehru Medical College, Wardha (M.S.).

\section{Materials and Methods}

The present study was conducted in the Department of Microbiology, Jawaharlal Nehru Medical College, Wardha (M.S). The period of study was 2 years from 1.9.2014 to 31.8.2016

\section{IEC clearance}

The study was approved by Institutional Ethical Committee. Study was a cross sectional A total number of 300 Klebsiella pneumoniae subspecies pneumoniae strains were isolated from different clinical samples, received from the indoor patients departments (IPD) of Acharya Vinoba Bhave Rural Hospital and Jawaharlal Nehru Medical College, Wardha (M.S) which is a tertiary care Hospital in rural setup. In the present study all 300 Klebsiella pneumoniae subspecies pneumoniae have been mentioned as Klebsiella pneumoniae.

The strains were characterized as Klebsiella pneumoniae according to conventional identification tests. (Washington et al., 2006) Klebsiella pneumoniae ATCC 700603, and Escherichia coli ATCC 25922 and Pseudomonas aeruginosa ATCC 27853 were 
used as control for all the conventional tests. All antibiotic disks and culture media used in this study were procured from Himedia Laboratories Pvt. Limited, India. Ethylene diamine tetraacetic acid (EDTA) and 3Aminophenylboronic acid (3-APB), Pheny boronic acid (PBA) were procured from Sigma-Alderich. All E test strips were procured from bioMereux.

All 300 Klebsiella pneumoniae strains were screened for Carbapenemase activity by Classical Hodge Test and Modified Hodge Test (MHT). Many phenotypic methods have been proposed by CLSI. The Classical Hodge test (Lee et al., 2003), Modified Hodge Test. (Lee et al., 2001) and Re-Modified Hodge test (Rai et al., 2011) are used for detection and confirmation of Carbapenemase activity. Double disc synergy test and disc potentiation test (Yong et al., 2002) using EDTA are based on this principle. For confirmation many other method used are MBL, E test using Imipenem, Imipenem-EDTA (Walsh et al., 2002) and reduction of MIC of carbapenems in presence of EDTA.

All 300 Klebsiella pneumoniae strains were tested for Metallobetalactamases (MBL) production by disc potentiation test and MBL$\mathrm{E}$ test strip (bioMerieux). The MBL-E test is considered as a standard Reference method for MBL detection. (Omair et al., 2012)

Detection of Klebsiella pneumoniae carbapenemases (KPC): producing strains was done by combined disc method. Using Imipenem and Phenyl boronic acid in combination the strains indicator used was Escherichia coli ATCC 25922.

\section{Classical Hodge test}

The indicator organism, Escherichia coli ATCC 25922 (turbidity adjusted to $0.5 \mathrm{Mc}$ Farland Standard) was used to inoculate the
Mueller Hinton agar plate as lawn culture and the test strain was heavily streaked. Then the plate was allowed to stand for 15 minutes at room temperature and a $10 \mu \mathrm{g}$ Imipenem disc (HiMedia) was placed at the center. The plate was incubated overnight at $37^{\circ} \mathrm{C}$. The presence of distorted inhibition zone was interpreted as a positive result for Carbapenem hydrolysis screening.

\section{Modified Hodge Test (MHT): (Jarlier et al., 1988)}

The broth culture of Escherichia coli ATCC 25922 the broth culture of Escherichia coli ATCC 25922. Four test strains of Klebsiella pneumoniae were streaked, the plate was incubated overnight at $37^{\circ} \mathrm{C}$. The presence of a cloverleaf shape zone of inhibition due to Carbapenemase production by the test strain was considered as positive.

\section{Imipenem- EDTA combined disc test for detection of $\mathrm{MBL}$}

In the combined disc test, if the increase in inhibition zone with the Imipenem and EDTA disc was $\geq 7 \mathrm{~mm}$ than the Imipenem disc alone, it was considered as MBL positive.

\section{MBL E-test}

MIC ratio of Imipenem/ Imipenem- EDTA (IP/IPI) of $\geq 8$ or deformation of ellipse or phantom zone indicate MBL.

Detection of Klebsiella pneumoniae carbapenemases (KPCs): (Tsakris et al., 2011)

KPCs can be mainly detected by combined disk method using Imipenem and Phenyl boronic acid in combination. Lawn culture of test strain (turbidity adjusted to $0.5 \mathrm{Mc}$ Farland) was put on Mueller Hinton (MH) agar and 2 Imipenem (10 $\mu \mathrm{g})$ discs were put 
widely apart. To one Imipenem disc Pheny boronic acid (PBA) solution was put, then the $\mathrm{MH}$ agar plates were incubated at $37^{\circ} \mathrm{C}$ overnight. After incubation, the diameter of growth inhibitory zone was compared between Imipenem disc with Phenyl boronic acid and Imipenem disc alone. The test should be considered positive when growth inhibitory zone around the disc containing Imipenem plus Phenyl boronic acid was $\geq 5 \mathrm{~mm}$ compared to zone diameter of Imipenem alone.

Detection of metallobetalactamase (MBL) and Klebsiella pneumoniae Carbapenemase (KPC): (Fattouh et al., 2015)

In this test, four discs of Imipenem $(10 \mu \mathrm{g})$ alone, Imipenem plus EDTA, Phenyl boronic acid and EDTA in combination were used. The production of both KPC and MBL were considered when the growth inhibitory zone diameter seen around Imipenem disc with both PBA+ EDTA had increased to $\geq 5 \mathrm{~mm}$, as compared to the growth inhibitory zone diameter seen around the Imipenem disc alone. When none of the three combined disc tests was positive, the isolate was considered negative for MBL and KPC production.

\section{Results and Discussion}

*In MBL and KPC Co-producer strains, four discs IPM/ IPM + EDTA, IPM+ PBA and IPM+EDTA+ PBA were used (Table 1).

Table 1 shows that out of 300 Klebsiella pneumoniae strains studied $88(29.3 \%)$ were Imipenem resistant and $212(70.7 \%)$ strains were Imipenem sensitive by Kirby- Bauer disc diffusion method as per CLSI guidelines 2016. All 300 Klebsiella pneumoniae strains were screened. for Carbapenem hydrolysis by Classical Hodge Test and Modified Hodge Test. Out of 88 Imipenem resistant strains 78 $(88.6 \%)$ were Classical Hodge Test positive and $82(93.2 \%)$ were Modified Hodge Test positive As confirmatory tests for MBL, Disc potentiation test and MBL E-Test were done. Out of 88 Imipenem resistant Klebsiella pneumoniae strains 46 (52.3\%) were positive by Disc potentiation test for MBL and those 46 were positive by MBL E-Test (Imipenem / Imipenam + EDTA i.e. IP/IPI) the confirmatory test for Klebsiella pneumoniae Carbapenemase (KPC) was done by combined disc method using Imipenem / Imipenem plus Phenylboronic acid. Out of 88 Imipenem resistant strains $39(44.3 \%)$ were positive for KPC by Combined disc methods. Out of 212 Imipenem sensitive strains $5(5 / 212$ i.e. $2.4 \%)$, 7 (3.3\%), 10 (4.7\%), $1(0.4 \%)$ and $11(5.2 \%)$ strains were positive by Classical Hodge test, Modified Hodge test, MBL confirmatory test (DP test and MBL E- test), KPC confirmatory test and Carbapenem hydrolysis positive due to $\mathrm{MBL}$ and $\mathrm{KPC}$ production respectively. Hence out of 88 imipenem resistant strains 85 (96.6\%) were positive for Carbapenemase production due to MBL and KPC. Out of 212 Imipenem sensitive strains, $11(5.2 \%)$ were positive for Carbapenem hydrolysis due to MBL and KPC production. Out of total 300 Klebsiella pneumoniae strains, 83 (27.7\%), 90 (30\%), 56 (18.7\%), 40 (13.3\%) and 96 (32\%) were positive by Classical Hodge test, Modified Hodge test, MBL confirmatory test, KPC confirmatory test and Carbapenem hydrolysis positive due to MBL and KPC production respectively.

Out of $40 \mathrm{KPC}$ producing strains $20(50 \%)$ strains produced KPC only but $19(47.5 \%)$ strains were MBL and KPC Co- producers and $1(2.5 \%)$ strain

Carbapenemases producing Klebsiella pneumoniae strains were detected by Classical Hodge test (CHT) and Modified Hodge test (MHT). The confirmatory tests for MBLs were done by Disc potentiation (DP) test and $\mathrm{E}$ test. In 2002, Walsh $\mathrm{T} \mathrm{R}$ et al., have 
reported that for detection of $\mathrm{MBL}$ producing strains MBL E test were $100 \%$ in agreement with results from PCR and biochemical methods. The confirmatory test for detection of KPC producing strains was done by combined disc method using Imipenem and Imipenem plus Phenyl boronic acid.

The Incidence of Carbapenem Resistant Klepsiella pneumoniae (CRKP) strains by various workers. In our present study, 29.3\% strains were CRKP. Datta et al., in 2012 reported 43.6\% CRKP strains from Delhi. (Datta et al., 2012) As many workers have reported that MBL and KPC genes can be carried by Carbapenem sensitive strains also and if only the Carbapenem resistant strains are studied, the MBL and KPC strains may be missed. Hence in the present study, all 300 Klebsiella pneumoniae strains were studied for detection of MBL and KPC. There were 88 (88/300 i.e. $29.3 \%$ ) Imipenem resistant strains but total Carbapenemase producing (MBL and KPC) strains were 85 (85/88 i.e. 97\%). Three (3/88 i.e. $3.4 \%)$ were Carbapenemase negative as these three strains were negative by Classical Hodge Test, Modified Hodge Test, and Confirmatory test also. Out of 212 Imipenem sensitive strains, 11 (5.2\%) strains were positive for Carbapenemase i.e. $10 \mathrm{MBL}$ and $1 \mathrm{KPC}$ producers were isolated from 212 Imipenem sensitive strains, but no MBL and KPC Co-producer was detected in these 11 strains. In the present study out of $39 \mathrm{KPC}$ producing strains $20(51.3 \%)$ strains produced KPC only and 19 (48.7\%) strains were MBL and KPC Co-producers. The MBL and KPC Co-producer strains were detected in the present study by using four discs IPM, IPM plus EDTA, IPM plus Phenyl boronic acid (PBA) and IPM plus EDTA plus Phenyl boronic acid (PBA). As EDTA inhibited MBL, comparing the zone diameter of IPM and IPM plus EDTA plus PBA, the strains producing MBL and KPC both were detected clearly.
In the present study, $18.7 \%$ Klebsiella pneumoniae strains were MBL producers. Singh et al., from North India have reported as low as $4.3 \%$ MBL producing strains. (Singh et al., 2015)

In the present study $13.3 \%$ KPC producing Klebsiella pneumoniae strains were isolated. Singh et al., in 2015 from North India reported quite high as $41.1 \%$ strains and lowest incidence of KPC (1.7\%) producing Klebsiella pneumoniae strains were reported by (Kumarasamy et al., 2010).

In the present study $(19 / 300$ i.e.6.3\%) Klebsiella pneumoniae strains were MBL and KPC Co- producers. In 2015, Singh et al., reported very high Incidence of $87.5 \% \mathrm{MBL}$ and KPC producing Klebsiella pneumoniae strains.

Figure 1 shows the Antibiotic Suseptibility profile of Klebsiella pneumoniae strains. The Highest sensitivity observed was Colistin (100\%) followed by Imipenem $70.6 \%$. The Klebsiella pneumoniae strains showed lowest sensitivity to Co-Trimoxazole (30.7\%) followed by Gentamicin 38.7\%. The highest resistant pattern observed was COT, GEN, CIP.

The present study was conducted to detect the incidence of Klebsiella pneumoniae subspp pneumoniae strains producing Carbapenemase.

A total number of 300 Klebsiella pneumoniae strains isolated from different clinical specimens in the Department of Microbiology, Jawaharlal Nehru Medical College, Wardha (M.S.) were studied. The clinical specimens were received from Indoor Patient Department (IPD) of different clinical specialities of Acharya Vinoba Bhave Rural Hospital and Jawaharlal Nehru Medical College, Wardha (M.S.). 
Table.1 Carbapenemase producing Klebsiella pneumoniae strains by various methods ( $\mathrm{n}=300)$

\begin{tabular}{|c|c|c|c|c|c|c|}
\hline \multirow[t]{3}{*}{$\begin{array}{l}\text { Klebsiella } \\
\text { pneumonia } \\
\text { n=300 }\end{array}$} & \multicolumn{2}{|c|}{$\begin{array}{l}\text { Screening test for } \\
\text { carbapenem } \\
\text { hydrolysis }\end{array}$} & \multicolumn{3}{|c|}{ Confirmatory test } & \multirow{3}{*}{$\begin{array}{c}\text { Total c } \\
\text { carbapenema } \\
\text { se producing } \\
\text { strains No. }\end{array}$} \\
\hline & $\begin{array}{l}\text { Classical } \\
\text { Hodge test } \\
\text { No. }\end{array}$ & $\begin{array}{c}\text { Modified } \\
\text { Hodge test } \\
\text { No. }\end{array}$ & \multicolumn{2}{|c|}{ MBL } & $\begin{array}{c}\text { KPC } \\
\text { (Combined } \\
\text { disc method) }\end{array}$ & \\
\hline & & & $\begin{array}{c}\text { DP Test } \\
\text { No. }\end{array}$ & $\begin{array}{c}\text { E-Test } \\
\text { No. }\end{array}$ & $\begin{array}{c}\text { IPM / IPM + } \\
\text { PBA No. }\end{array}$ & \\
\hline $\begin{array}{c}\text { Imipenem } \\
\text { Resistant } \\
\mathrm{n}=88 \\
\end{array}$ & 78 & 82 & 46 & 46 & $39^{*}$ & 85 \\
\hline $\begin{array}{c}\text { Imipenem } \\
\text { sensitive } n= \\
212 \\
\end{array}$ & 5 & 7 & 10 & 10 & 1 & 11 \\
\hline $\begin{array}{c}\text { Total } \\
\mathbf{n}=\mathbf{3 0 0}\end{array}$ & 83 & 90 & 56 & 56 & 40 & 96 \\
\hline
\end{tabular}

Fig.1 The Antibiotic susceptibility profile of Klebsiella pneumoniae strains ( $\mathrm{n}=300)$

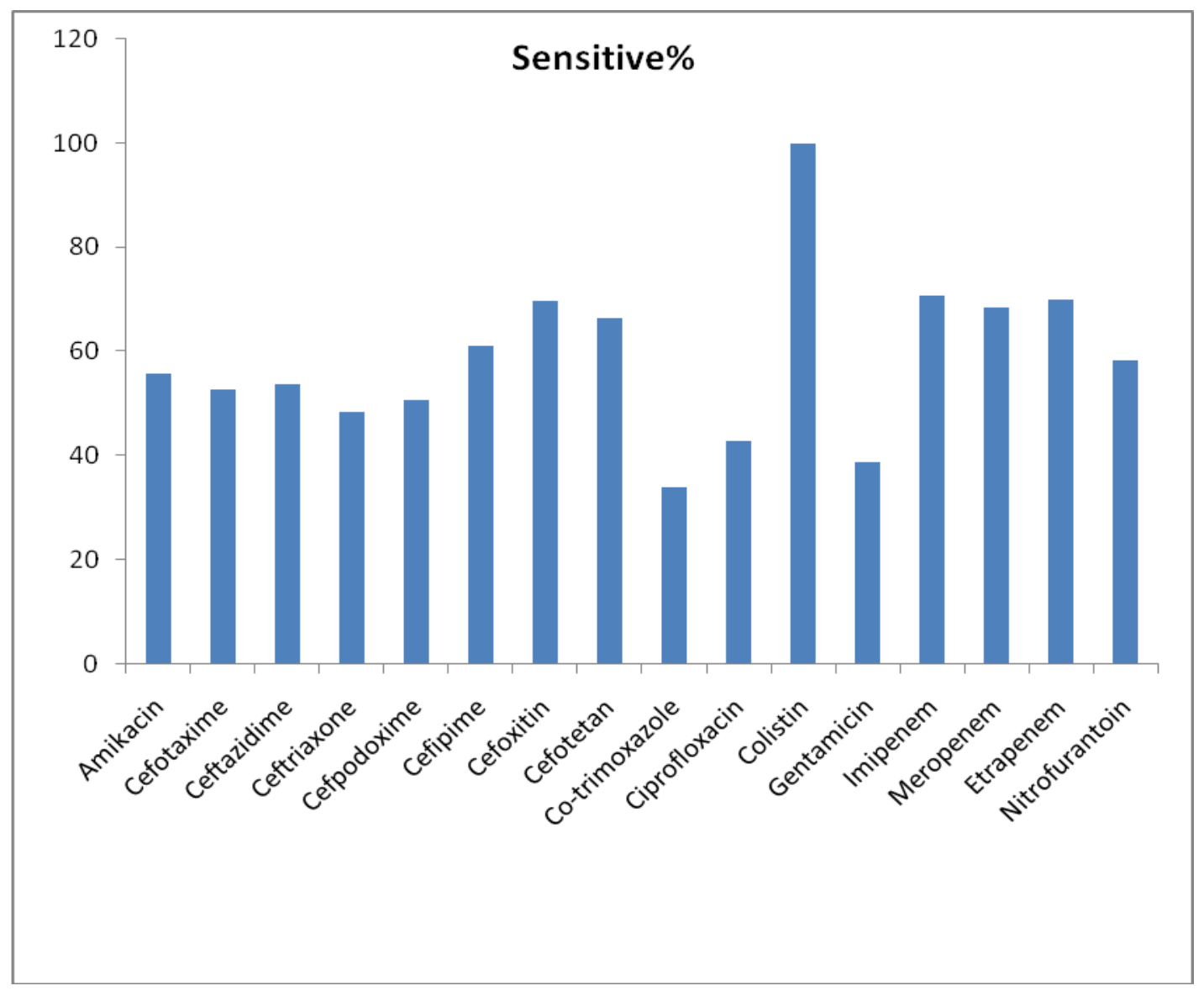


The rising trend of developing resistance to multiple antibiotics in microorganisms leads to therapeutic failure. Presently antimicrobial resistance is a major threat to patient care and infectious disease control worldwide.

Klebsiella pneumoniae strains can also produce Carbapenemase. Such as Metallobetalactamases (MBLs) and Klebsiella pneumoniae carbapenemases (KPCs) confer resistance to $3^{\text {rd }}$ generation Cephalosporines and Cephamycins etc. which are commonly used for treating patients. Carbapenems are used as a last resort in Carbapenemase producing Klebsiella pneumoniae strains and other Enterobacteriaceae and Gram negative bacteria. Carbapenemases such as MBL and KPC producing strains develop resistance to $3^{\text {rd }}$ generation Cephalosporines, Cephamycins and Carbapenems etc. The major problem for Carbapenemase producing strains are, therapeutically available inhibitors are not available and rapid dissemination can occur to other Gram negative bacteria e.g. Escherichia coli, Pseudomonas aeruginosa, Acinetobacter baumanii etc. in any Health Care set up. Hence, detection of Carbapenemases such as MBL and KPC producing strains are very important for prompt reporting of these strains to clinicians for effective treatment and to take proper infection control measures, to prevent dissemination of these strains in Health care set up.

The present study was conducted in the Department of Microbiology, Jawaharlal Nehru Medical College, Wardha (M.S.). A total number of 300 Klebsiella pneumoniae strains were isolated from different clinical specimens like urine, blood, pus and wound swab etc. and were identified by conventional methods.

The confirmatory phenotypic tests are simple to perform and quite cheap. For detection of Carbapenemase production the $\mathrm{E}$ tests are standard reference methods and molecular method like Polymerase chain reaction (PCR) is a gold standard, but both are costly require expertise. Moreover PCR requires tailor made primers and cannot detect the variants.

Hence, to conclude, Klebsiella pneumoniae strains which are one of the most common isolates from different clinical specimens must be tested for detection of Carbapenemase by confirmatory phenotypic tests like combined disc methods for KPC and Disc potentiation tests for MBL by Clinical Microbiology Laboratory to prevent the delay in detection of these Carbapenemase producing strains to get a good therapeutic outcome for the patients and to prevent the spread of these $\beta$-lactamases producing strains in the Health care set up by taking proper Infection control measures.

\section{References}

Datta P, Gupta V, Shivani G. Phenotypic method for differentiation of carbapenemases in Enterobacteriaceae. Study from North India. Indian J Pathol Microbiol. 2012; 55(3): 357-360.

Fattouh M, Nasr El- din A, Omar² Mohamed A. Detection of Klebsiella pneumonia Carbapenemase (KPC) producing Gram Negative Superbugs: An Emerging cause of Multidrug-resistant Infection in General surgery Department of Sohag University Hospital, Egypt. Int. J. Curr. Microbiol. App. Sci. 2015; 4(5): 1-15.

Jarlier $\mathrm{V}$ et al., extended spectrum betalactamase conferring resistance to newer $\quad \beta$-lactam agents in Enterobacteriaceae: Hospital prevalence and susceptibility patterns. Rev Infect Dis. 1988; 10: 867-878.

Kumarasamy K K, Toleman M A, Walsh T R, Bagaria J, Butt F, Balakrishnan R et al., Emergence of a new antibiotics resistance mechanism in India, Pakistan 
and the U.K. A molecular, biological and epidemiological study. Lancet mInfect Dis. 2010; 10: 597-602.

Lee K, Chong Y, Shin H B, Shin Y A, Kim $\mathrm{D}$, Yong, Yum $\mathrm{J} \mathrm{H}$. Modified Hodge and EDTA disc synergy test to screen metallo beta-lactamases producing strains of Pseudomonas spp. and Acinetobacter spp. Clin Microbiol Infect. 2001; 7: 88-91.

Lee K, Lim Y S, Yong D et al., Evalution of Hodge test and Imipenem-EDTA double disc synergy test for differentiation of metallo betalactamases producing clinical isolates of Pseudomonas spp. and Acinetobacter spp. J Clin Microbiol. 2003; 41: 4623 4629.

Omair M, Usman J, Kaleem $\mathrm{F}$ et al., Evalution of Combined disc methods for detection of metallo- betalactamase producing Gram negative bacilli. Malaysian Jr. of Microbiol. 2012: 8 (1): 21-25.

Queenan A M, Bush K. Carbapenemase: the versatile $\beta$-lactamases. Clin Microbiol Rev. 2007; 20: 440-458.

Rai S, Manchanda V, Sigh N P et al., Zinc dependent carbapenemases in clinical isolates of family Enterobacteriaceae. Indian J. of Medical Microbiol. 2011; 29(3): 275-279.

Singh M, Kakati B, Agrarwal R K, Aarti Kotwal. Detection of Klebsiella pneumoniae Carbapenemases (KPC) among ESBL/ MBL producing Clinical Isolates of Klebsiella pneumonia. International Journal of Current Microbiology and applied Sciences 2015; 4(4): 726-731.

Tsakris A, Digalaki K T, Poulou A et al., Comparative evaluation of combined disc tests using different boronic acid compounds for detection of Klebsiella pneumoniae carbapenemase- producing Enterobacteriaceae clinical isolates. J Clin Microbiol. 2011; 49(8): 28042809.

Walsh T R, Bolmstrom A, Qwarnstrom A et al., Evolution of new $\mathrm{E}$ test for detecting metallo betalactamases in routine clinical testing. J Clin Microbiol. 2002; 40: 2755-2759.

Walther-Rasmuseen J, Hoiby N. OXA- type Carbapenemases. Journal Antimicrob Chemothers. 2006; 57: 373-378.

Washington C W Jr, Stephen DA, Williams M J, Elmer W K, Gary W P, Paul C S, Gail L W. Enterobacteriaceae.in chapter 6. Koneman's Color Atlas and Textbook of Diagnostic Microbiology, $6^{\text {th }}$ ed, Lippincott Williams \& Wilkins, Philadelphia, USA, 2006; pg.211-302.

Yong D, Lee K, Yum J H, Shin H B, Rossolini G M, Chong Y. ImipenemEDTA Disc method for differentiation of metallo betalactamases producing clinical isolates of Pseudomonas spp. and Acinetobacter spp. J Clin Microbiol. 2002; 40: 3798-3801.

\section{How to cite this article:}

Priyanka Singh. 2018. The Incidence of Carbapenem Resistance in Klebsiella pneumoniae Subspecies pneumoniae (CRKP) and Antibiotic Susceptibility Profile of Klebsiella pneumoniae Subspecies pneumoniae strains. Int.J.Curr.Microbiol.App.Sci. 7(01): 594-601. doi: https://doi.org/10.20546/ijcmas.2018.701.072 